Für unser modernes Labor in Kopenhagen suchen wir

\title{
eine(n) Metallographin(en)
}

mit 2-4 Jahren Berufserfahrung als Metallograph oder Werkstoffprüfer.

Unser neuer Mitarbeiter sollte dazu bereit sein, sowohl selbständig als auch im Team aktiv mitzuarbeiten.

Als wesentliche Aufgaben stellen wir uns vor:

- optimale Präparationsmethoden für verschiedene Materialien auszuarbeiten

- zur Entwicklung neuer Verbrauchsmaterialien und Geräte beizutragen

- bei Kursen und Kundenbesuchen beratend mitzuwirken

Nach der Einarbeitungszeit kommen auch verkaufsfördernde Aktivitäten im Ausland in Frage.

Fremdsprachenkenntnisse, z. B. Englisch, sind von Vorteil, da unsere Arbeit in einer internationalen, offenen Atmosphäre stattfindet.

Wenn Sie Interesse an dieser Position haben, senden Sie uns bitte bis spätestens 1 . September 1984 Ihre Bewerbungsunterlagen unter Angabe des frühest möglichen Einstellungstermins zu.

STRUERS A/S

Abteilung $M$

z. Hd. Herrn R. Hoeg

Valhøjs Allé 176

DK-2610 Rødovre/Kopenhagen

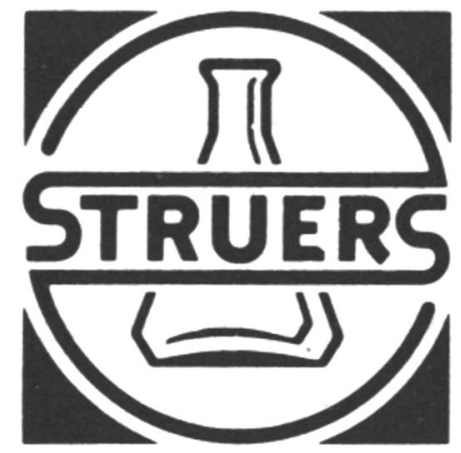



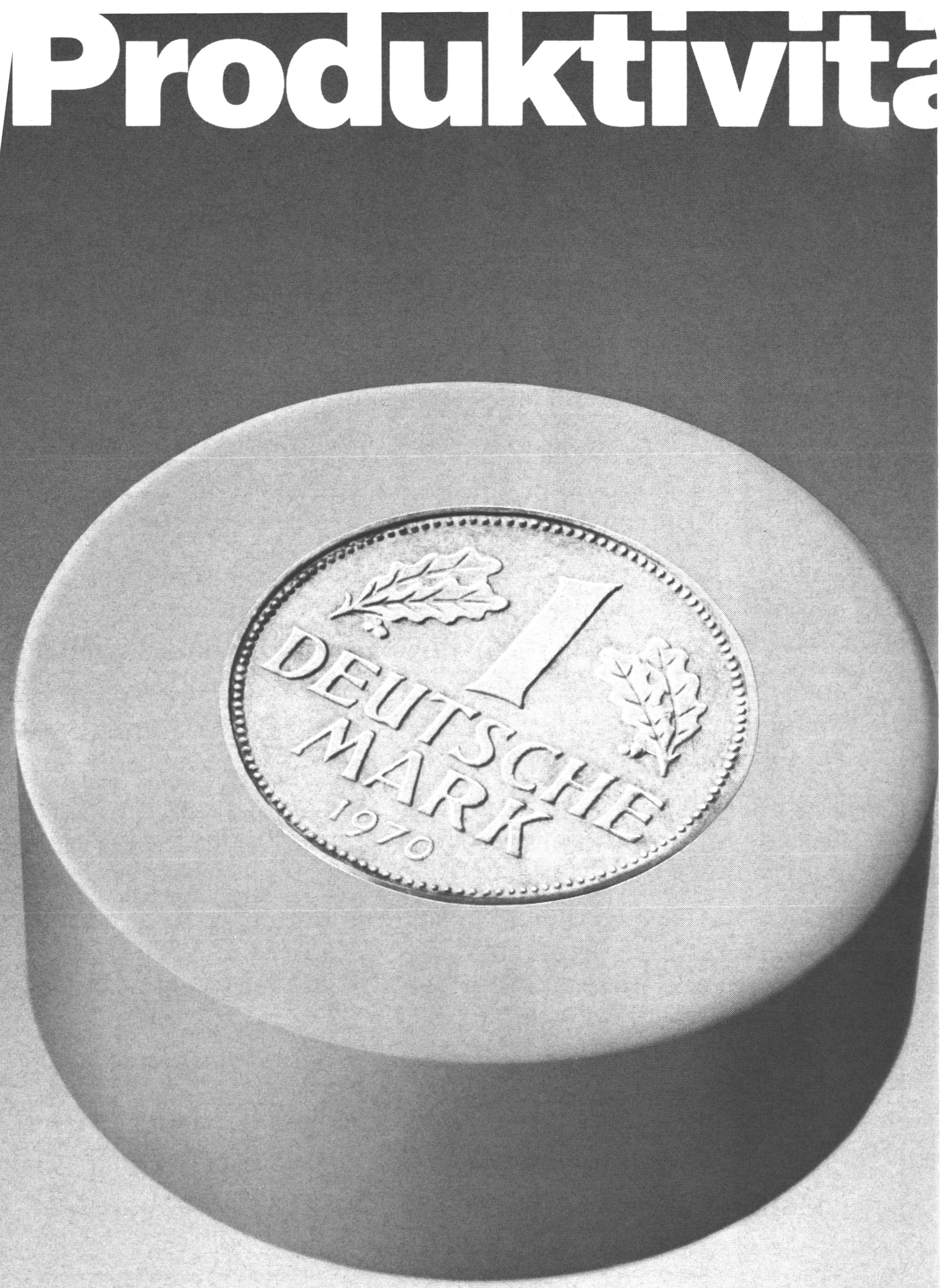


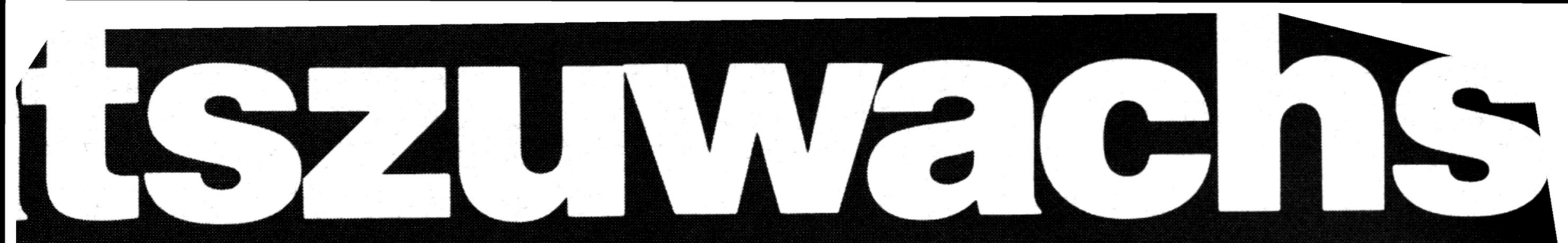

Schnellere und wirtschaftlichere Materialprüfung mit verbesserten Ergebnissen - ein "magisches Dreieck“, das Wunschtraum bleiben muß? Das große Materialprüfungsprogramm von Kulzer beweist, wie Produktivität im quantitativen und qualitativen Sinne wachsen kann: Einbetten, Schleifen und Polieren - von bisher langer Dauer - lassen sich auf Minuten verkürzen. Produktivitätszuwachs von Kulzer - Vorteile, die Ihre Kosten senken.

\section{Kulzer hat das große Programm tur die Materialprüfung}

Technovit 4000 - $4004-4071$ kaltpolymerisierende Kunststoffe, für spaltfreie transparente - schnelle Einbettungen, mit ausgezeichneter Randschärfe.

Technovit sooo für Schlifeinbettungen für REM. Mikrosonde und zum elektrolytischen Wischpolleren. Aushärtung in 7 Whuten. Fur eintache, schnelle, sichere Veratbelung:

Uhuer.al robenhalter zum Einbetten und zur Aumahme von bis zu 6 Proben in einem Arbeitsgang. Rationell: schliffertic im Probenhalter in 6-7 Minuten.

Technotron Schleif- und Poliersystem arbeitet schnell, einfach und wirtschaft lich - nur ein Schleif- und ein Poliervor gang mit hervorragenden Ergebnissen.

Bitte fordern Sie ausführliche Informatio nen an.

Kulzer \& $\mathrm{Co} \mathrm{GmbH}$, Bereich Technik Postfach 1320, D-6382 Friedrichsdorf 1 Telefon 06172/732-0, Telex $415863 \mathrm{Kulzd}$

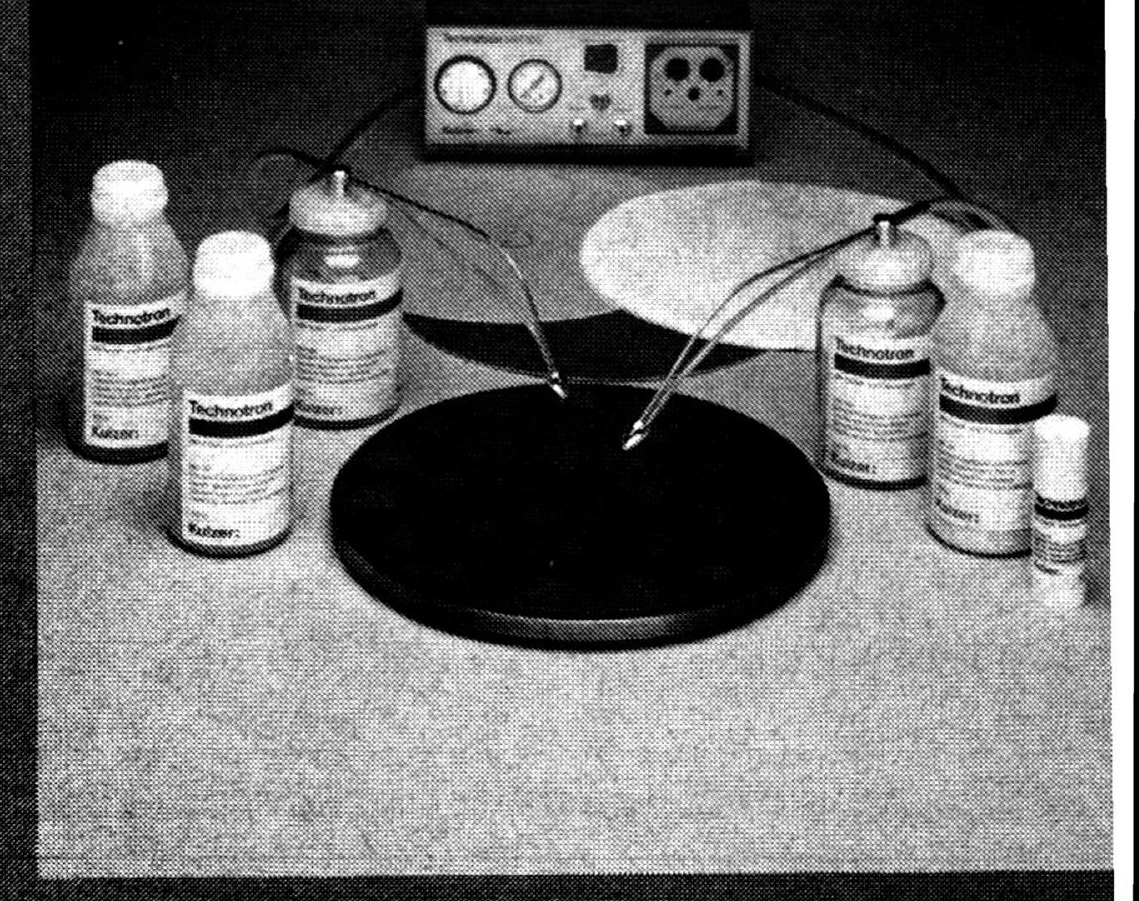


Praktische Metallographie

Herausgeber/Editor

\author{
Hauptschriftleiter/Editor in Chief
}

\section{Redaktionssekretariat/Secretary}

\section{Schriftleitung/Editorial Board}

Prof. Dr. H.-E. Bühler

Didier-Werke AG, Lessingstraße 16-18

D-6200 Wiesbaden

\section{Chris Bagnall}

Westinghouse Electric Corporation

Advanced Reactor Division

P. O. Box 158

Madison, PA 15663, USA

Dr. J. L. Chermant

Equipe Matériaux-Microstructure, L. A. 251

I.S.M.R.A.-Université, F-14032 Caen Cedex

Dr. G. Elssner

Max-Planck-Institut für Metallforschung

Seestraße 92, D-7000 Stuttgart 1

R. J. Gray

National Laboratory Oak Ridge

Oak Ridge, Tenn. 37830, USA

Prof. Dr. E. Hornbogen

Ruhr -Universität, Bochum

Postfach 2148, D-4630 Bochum

Dr. H. P. Hougardy

Max-Planck-Institut für Eisenforschung

Max-Planck-Straße 1, D-4000 Düsseldorf

Prof. Dr. F. Jeglitsch

Montanuniversität

A-8700 Leoben

Verlag und Anzeigenannahme/

Publisher and

Advertisement Contractor

Druck/Printing

Erscheinungsweise monatlich

Jährlicher Bezugspreis 1984 DM 139,-*

Einzelheft DM 16,- ${ }^{\star}$ zzgl. Porto

Ohne schriftliche Genehmigung des Verlages dürfen weder Nachdruck, Ubersetzungen, Photokopie, Mikrofilm noch Ubernahme in Datenverarbeitungsanlagen bzw. deren Sprachen oder Informationssysteme irgendwelcher Art erfolgen. Fotomech. Vervielfält. nach Maßgabe des zw. dem Börsenv. des Dt. Buchhandels e. V. und dem Bundesverband der Dt. Industrie abgeschl. Rahmenabkommens gestattet. Abbestellungen nur bis spätestens 2 Mon. vor Ablauf des Kalender-Jahres.

In Zusammenarbeit mit der DGM.

\section{Practical Metallography}

Prof. Dr. G. Petzow

Max-Planck-Institut für Metallforschung Heisenbergstraße 5, D-7000 Stuttgart 80

Dr. H. E. Exner

Max-Planck-Institut für Metallforschung

Seestraße 92, D-7000 Stuttgart 1

Karin Exner

Amselweg 3, D-7250 Leonberg 7

Prof. Dr. Ursula Martius Franklin

University of Toronto

Toronto 5, Ontario, Canada

J. A. Nelson

Buehler Ltd.

41 Waukegan Road, Lake Bluff, IL 60044, USA

Prof. Dr. G. Ondracek

Universität und Kernforschungszentrum Karlsruhe

Postfach 36 40, D-7500 Karlsruhe

Dr. L. Samuels

Materials Research Labs.

POB 50, Ascot Vale, Victoria, Australia

Dr. H.-J. Schüller

Allianz-Zentrum für Technik $\mathrm{GmbH}$

Krausstraße 14, D-8045 Ismaning

Prof. Dr. Stickler

Universität Wien

Währingerstraße 42, A-1090 Wien

Fachstudiendirektorin Charlotte Wachau

Lette-Verein

Fabeckstraße 43, D-1000 Berlin 33

\section{Dr. Riederer-Verlag GmbH \\ Postfach 447}

D-7000 Stuttgart 1, Telefon (07 11) 639797

Badendruck GmbH, Karlsruhe

Issued monthly

Annual subscription rate 1984 DM 139,-* Single copy DM 16,-" plus delivery charges

All rights reserved. Without permissions in writing by the publisher neither reprints, translations, photoprints, microfilms, nor transmitting in computer-systems or Informationcenters in any way will be allowed. Reproduction by photography or xerox etc. for private use only. Cancellations of subscriptions not later than 2 months to the end of each current year.

In cooperation with the German Society for Metals 